\title{
Exploring the Transferability of Large Supramolecular Assemblies to the Vacuum-Solid Interface
}

\author{
Wei $\mathrm{Xu}^{1, \S}$, Mingdong Dong ${ }^{1}$, Henkjan Gersen ${ }^{1, \#}$, Socorro Vázquez-Campos ${ }^{2}$, Xavier Bouju ${ }^{3}$, Erik Lægsgaard ${ }^{1}$, \\ Ivan Stensgaard ${ }^{1}$, Mercedes Crego-Calama ${ }^{2, \dagger}$, David N. Reinhoudt ${ }^{2}$, Trolle R. Linderoth ${ }^{1}(\varangle)$, and Flemming \\ Besenbacher $^{1}(\square)$ \\ Interdisciplinary Nanoscience Center (iNANO) and Department of Physics and Astronomy, Aarhus University, Ny Munkegade, \\ 8000 Aarhus C, Denmark \\ 2 Laboratory of Supramolecular Chemistry and Technology, Materials Science and Technology of Polymers, MESA ${ }^{+}$Institute for \\ Nanotechnology, University of Twente, P.O. Box 217, 7500 AE Enschede, The Netherlands \\ Nanoscience group, CEMES-CNRS, 29 rue Jeanne Marvig, 31055 Toulouse, France \\ $\S$ Current address: Departments of Chemistry and Physics, The Pennsylvania State University, University Park, Pennsylvania \\ 16802, USA \\ " Current address: Nanophysics and Soft Matter Group, Department of Physics, University of Bristol, Tyndall Avenue, BS8 1TL \\ Bristol, UK \\ Current address: Holst Center (IMEC-NL), High Tech Campus 48, 5656 AE Eindhoven, The Netherlands
}

Received: 1 April 2009 / Revised: 1 May 2009 / Accepted: 1 May 2009

(CTsinghua University Press and Springer-Verlag 2009. This article is published with open access at Springerlink.com

\begin{abstract}
We present an interplay of high-resolution scanning tunneling microscopy imaging and the corresponding theoretical calculations based on elastic scattering quantum chemistry techniques of the adsorption of a goldfunctionalized rosette assembly and its building blocks on a $\mathrm{Au}(111)$ surface with the goal of exploring how to fabricate functional 3-D molecular nanostructures on surfaces. The supramolecular rosette assembly stabilized by multiple hydrogen bonds has been sublimed onto the $\mathrm{Au}(111)$ surface under ultra-high vacuum conditions; the resulting surface nanostructures are distinctly different from those formed by the individual molecular building blocks of the rosette assembly, suggesting that the assembly itself can be transferred intact to the surface by in situ thermal sublimation. This unanticipated result will open up new perspectives for growth of complex 3-D supramolecular nanostructures at the vacuum-solid interface.
\end{abstract}

\section{KEYWORDS}

Self-assembly, surface nanostructures, scanning tunneling microscopy, supramolecular assembly, hydrogen bonding

\section{Introduction}

Self-assembly of molecular building blocks into supramolecular architectures has played an important role in creating 2-D surface nanostructures [1-16]. However, an important step for the rational design

Address correspondence to Trolle R. Linderoth, trolle@inano.dk; Flemming Besenbacher, fbe@inano.dk 
of functional molecular devices on surfaces is to extend these nanostructures in the third dimension. One strategy to achieve this goal would be to directly transfer the knowledge of supramolecular chemistry in solution to 2-D solid surfaces. However, it is generally expected that on-surface self-assembly processes may be completely different from the corresponding self-assembly processes in solution. With respect to the on-surface self-assembly, several factors such as steric effects, adsorbate-substrate registry, and surface electronic effects may potentially influence the selfassembly processes to deviate from the equilibrium conditions. This may result in kinetically trapped nanostructures that are very different from the selfassembled products in solution [1, 2, 11, 12]. Thus, a more viable strategy to pattern surfaces by 3-D nanostructures would be to directly transfer the welldefined 3-D assemblies achieved by supramolecular synthesis in solution to a 2-D solid interface.

Recently, an interesting 3-D self-assembled supramolecular compound, the so-called rosette assembly as shown in Scheme 1, has been synthesized in solution and characterized in great detail [17-21]. The rosette assembly M2 is built up from relatively big building blocks in the form of the calix[4]arene dimelamine derivative M1 and smaller building blocks in the form of diethylbarbituric acid (DEB). The self-assembly of M2 involves the formation of 36 complementary hydrogen bonds between three M1 and six DEB units in an organic solvent. It has been demonstrated that the $\mathrm{M} 2$ rosette assembly has a well-defined 3-D shape and size provided by the complementary hydrogen bonds. One big advantage of this rosette assembly is that even slight modifications in the structure of the building blocks yield different functionalized rosette assemblies. Therefore, a variety of such assemblies can be generated by chemical modifications of the building blocks. For instance, gold-functionalized rosette assemblies have been synthesized, allowing gold atoms to be aligned on surfaces. In this work, we have investigated the adsorption of the goldfunctionalized rosette assembly (M2) and its building blocks (M1 and DEB) on a $\mathrm{Au}(111)$ surface by scanning tunneling microscopy (STM) under ultrahigh vacuum (UHV) conditions with the goal to explore how to fabricate functional 3-D molecular nanostructures on surfaces.

\section{Results and discussion}

Prior to the study of the rosette assembly M2 we
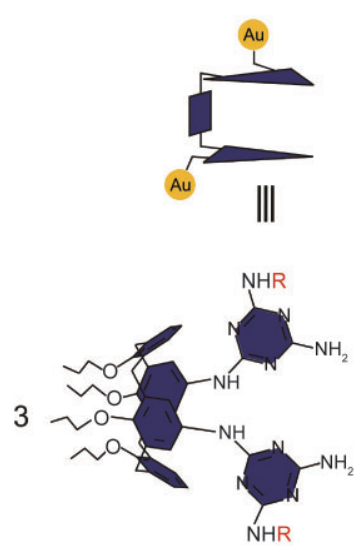

M1

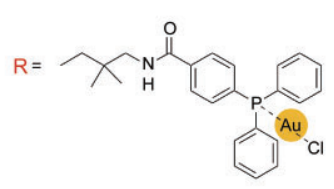

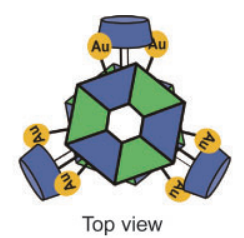

| |

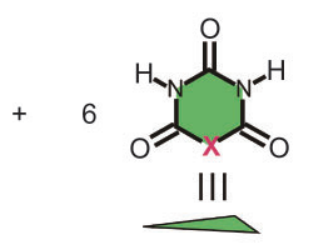

DEB: $\mathrm{X}=\mathrm{C}\left(\mathrm{CH}_{2} \mathrm{CH}_{3}\right)_{2}$

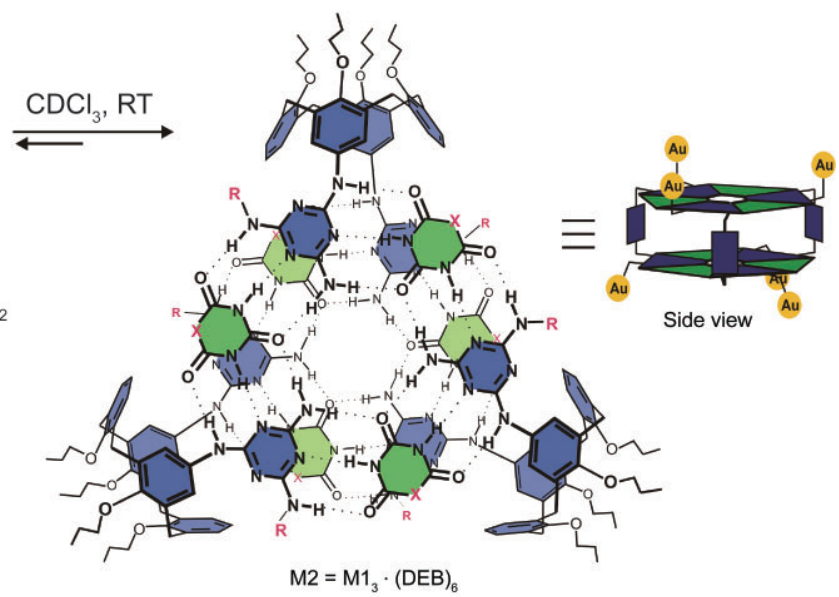

Scheme 1 Schematic representation of the structures of M1, DEB, and the formation of the rosette assembly M2 (RT representsç room temperature) 
investigated how the individual building blocks self-assemble on $\mathrm{Au}(111)$. After deposition of DEB molecules, large molecular islands with well-ordered structure were observed on the $\mathrm{Au}(111)$ surface as depicted in Fig. 1(a) [21]. This self-assembled structure consists of DEB molecules forming molecular chains with zigzag arrangements, and each bright spot in the STM image corresponds to one single DEB molecule. The self-assembled DEB structure can be described as 1-D molecular chains formed by hydrogen bonds, which are subsequently linked together through their alkyl chains via van der Waals (vdW) interactions to form the 2-D ordered structure. This characteristic bonding configuration is also reflected in the high-resolution STM images, as between every molecular chain a fairly big gap is observed in which the interlocked alkyl chains are visible [21].

After deposition of the M1 building block, a wellordered lamella structure was formed, as depicted in Fig. 1(b) [20]. Notice that in this case we have studied a modified M1 compound containing two coordinated gold atoms as illustrated in the inset of Fig. 1(b). Moreover, as depicted in high-resolution STM images (Fig. 1(b)), the individual M1 building block is imaged as one bright central protrusion linked to the two "arms" on both sides. The bright features at the ends of the two arms are ascribed to the coordinated gold atoms. Due to the well-defined 3-D conformation of the M1 building block, a non-flat
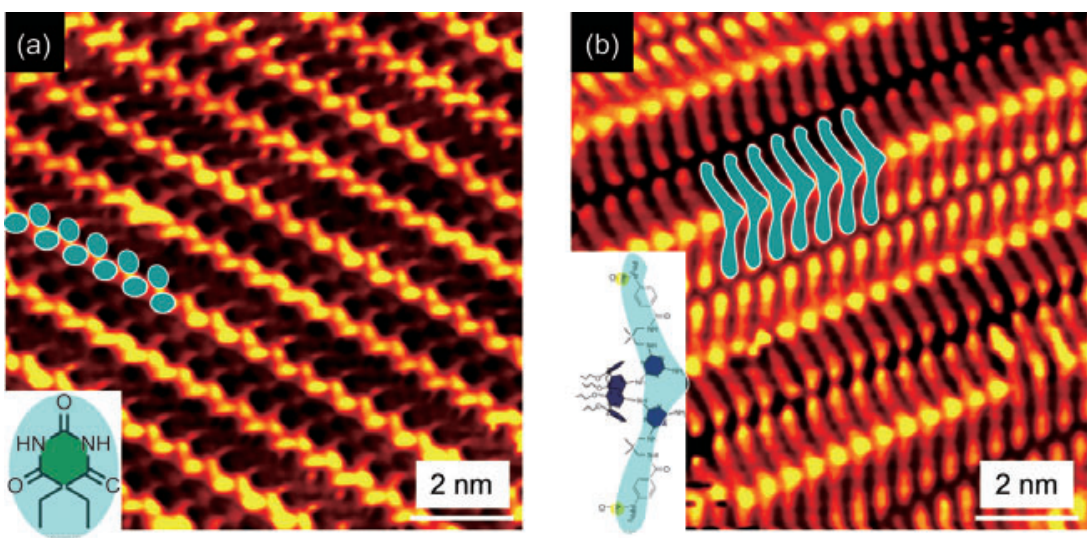

Figure 1 High-resolution STM images of the self-assembled nanostructures of DEB (a) and $\mathrm{M} 1$ (b). $I_{\mathrm{t}}=-0.5 \mathrm{nA}, V_{\mathrm{t}}=-1250 \mathrm{mV}, I_{\mathrm{t}}=-0.6 \mathrm{nA}, V_{\mathrm{t}}=-880 \mathrm{mV}$ for (a) and (b), respectively. The schematic drawings superimposed in each image illustrate how the molecules are arranged within the nanostructures (each protrusion corresponds to an individual DEB and M1 molecule, respectively) geometry is anticipated upon adsorption of M1 on the surface. This is consistent with the STM images, where the apparent heights of the two "arms" on either side of the central protrusion are clearly seen to be different. From these observations we conclude that the lamella structure is associated with the intact M1 building block [20].

After characterization of the individual building blocks (DEB and M1), we continued to explore the complete rosette assembly. The M2 complex was thermally sublimed in situ from a glass crucible heated to $380 \mathrm{~K}$ (see the Experimental section). After this procedure we observed large well-ordered islands on the $\mathrm{Au}(111)$ surface as shown in Figs. 2(a) and 2(c). The islands consist of periodic double rows of protrusions, as is particularly visible in the large-scale STM image in Fig. 2(c). The close-up STM image in Fig. 2(a) shows subtle differences in the imaged shape of the features in adjacent double rows, and therefore in the direction perpendicular to the rows the structure only repeats itself for every second row as indicated by the green lines in Fig. 2(a). The structure exhibits a number of characteristic point defects where individual entities in the double rows are missing. In images acquired at high resolution (Fig. 2(a)) it appears that there are molecular features even inside these point defects, suggesting that the structure consists of a double or even multilayer of molecular entities. Evidently, the structure observed after deposition of M2 is entirely different from the ones formed by the M1 and DEB building blocks alone on the $\mathrm{Au}(111)$ surface (as seen by comparison with Fig. 1). This shows that thermal sublimation of the hydrogen bonded M2 assembly does not lead to fragmentation of the complex and deposition of the individual molecular building blocks which subsequently phase segregate on the surface. In further experiments where DEB and M1 were co-deposited on the surface, we were not able to synthesize the observed well-ordered structure associated with the deposition of the 
M2 assembly. This shows that the structure observed in Figs. 2(a) and 2(c) is not the result of surface selfassembly of individual building blocks formed by thermal fragmentation of the M2 assembly. Taken together, these observations strongly suggest that the structural integrity of the M2 compound is at least partially sustained during thermal sublimation and the structure observed after deposition of the M2 compound retains a "memory" of the structure of the M2 assembly itself. Additionally, there are striking quantitative similarities between the repeat distances of the structure formed after deposition of M2 and the dimensions of the rosette assembly M2 itself (Fig. 2(b)). The unit cell of the periodic structure indicated in Fig. 2(a), has side lengths of $2.5 \mathrm{~nm} \pm 0.2 \mathrm{~nm}$ and $1.5 \mathrm{~nm} \pm 0.2 \mathrm{~nm}$, which compare extremely well with the $\sim 2.8 \mathrm{~nm}$ edge length and $\sim 1.3 \mathrm{~nm}$ thickness, respectively, of the rosette assembly. We therefore tentatively propose that the M2 rosette assemblies are in fact deposited intact on the surface, where they align in an "edge-on" manner as schematically illustrated in Fig. 2(a).

A line scan (see Fig. 2(d)) at the perimeter of the self-assembled molecular island, obtained from the constant current image depicted in Fig. 2(c), shows an apparent height of only $\sim 0.3 \mathrm{~nm}$ compared to

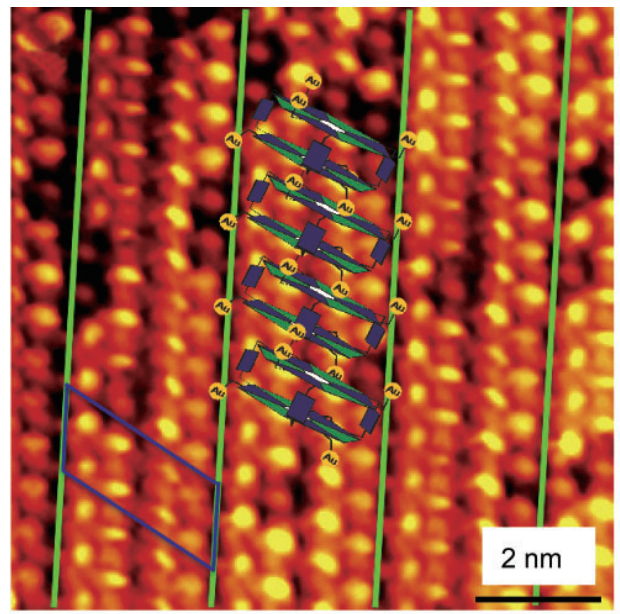

(a)

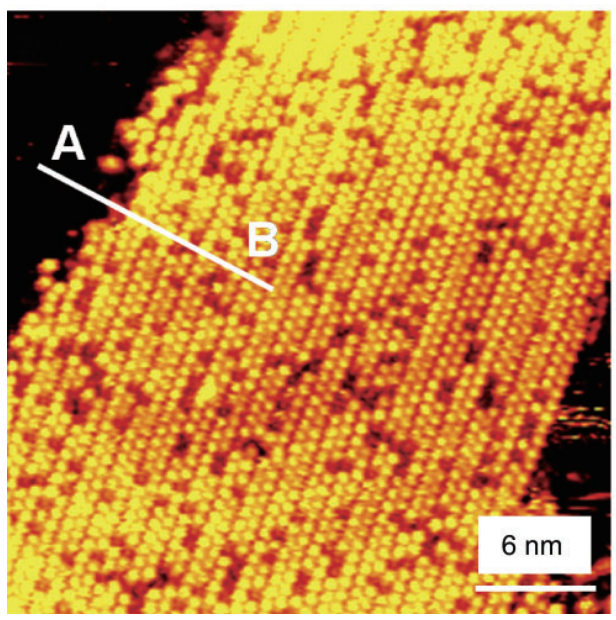

(c)
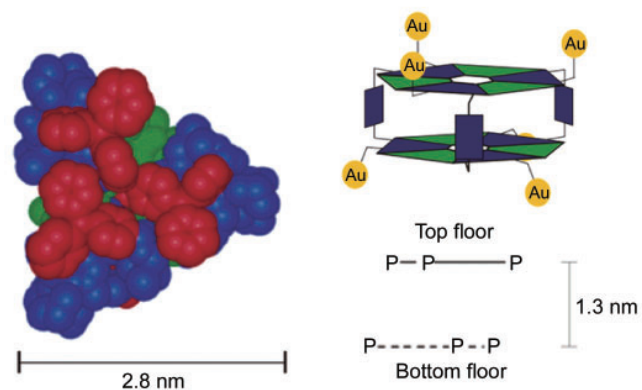

(b)

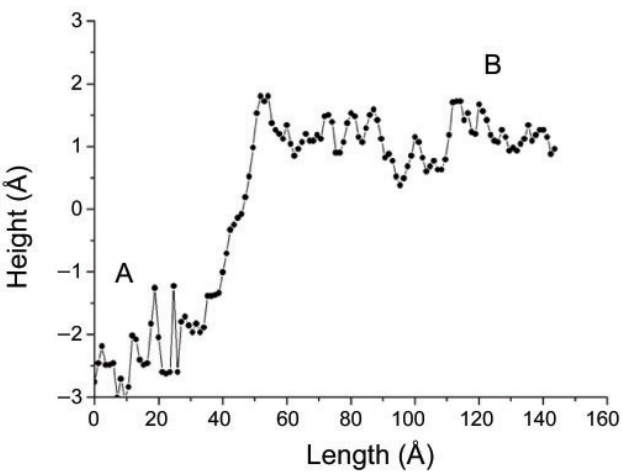

(d)

Figure 2 (a) STM image showing the surface molecular nanostructure associated with deposition of M2. (b) Left side: top view of the molecular structure of the double rosette $\mathrm{M}_{3} \cdot(\mathrm{DEB})_{6}$ obtained from molecular modeling using a gas phase energy-minimization routine. The calix[4]arene dimelamine framework is shown in blue, the triphenylphosphane groups in red, and the DEB moieties in green. Right side: schematic side view of the $\mathrm{M}_{3} \cdot(\mathrm{DEB})_{6}$ complex. (c) Large-scale image of the structure associated with deposition of $\mathrm{M} 2$. (d) Height profile taken along the line indicated in (c). $I_{\mathrm{t}}=-0.4 \mathrm{nA}, V_{\mathrm{t}}=-1250 \mathrm{mV}$ 
the surrounding $\mathrm{Au}(111)$ substrate, i.e., significantly smaller than the physical height of the rosette assembly M2 in the proposed edge-on adsorption geometry. Agreement between physical and apparent height is, however, not generally expected in constant current STM images, where the contrast represents the required Z-movement of the STM tip to maintain the demanded tunnel current, and therefore mainly depends on the electronic structure of the surface and the transmission coefficient for electron transport through the molecular overlayer. Numerous studies of self-assembled monolayers (SAMs) of alkanethiols on gold surfaces have reported apparent heights much lower than the alkane thickness reflecting the low conductivities of the molecular films [22-24]. To obtain further insight into STM imaging of the M2 complex, we have performed theoretical calculations using elastic scattering quantum chemistry (ESQCSTM) image techniques and extended semi-empirical atom superposition and electron delocalization (ASED+) codes [25-27]. As the entire M2 assembly is prohibitively large for efficient modeling, we focus instead on a single M1 building block since we expect most of the tunnel current to go through M1 when the M2 assembly is adsorbed as proposed in the model superimposed on Fig. 2(a). The structure of the simulated system (see the inset in Fig. 3) is rather similar to a single M1 unit extracted from an M2 complex embedded in the molecular crystal. In the calculation, the coordinated gold atom closest to the surface was kept at a height of $0.56 \mathrm{~nm}$ and the other at $1.72 \mathrm{~nm}$ resulting in a total height of the adsorbed M1 molecule of $2.21 \mathrm{~nm}$. A calculated line scan over the M1 molecule adsorbed on the gold surface is represented in Fig. 3, and shows protrusions with heights between 0.4 and $0.7 \mathrm{~nm}$. The STM image calculation was performed at a lower tunnel current than that used in the experiment since otherwise the tip and molecule would have collided and it would not have been possible to trace the contour of the molecule.
We therefore conclude that a 3-D object such as the rosette assembly, exhibiting low conductivity, and a low electronic coupling with the surface, has a very small transmission coefficient for electron tunneling through it [28], in agreement with the observed low apparent height for the molecular island in Fig. 2(c). We note, however, that the experimentally observed apparent height $(\sim 0.3 \mathrm{~nm})$ in combination with the assumed edge-on adsorption configuration of the large rosette assemblies implies that the outermost apex atoms of the STM tip could have protruded inside the molecular film during scanning of the STM images. That it is possible to obtain such relatively low-noise STM images under these conditions must qualitatively be ascribed to conformational flexibility in the molecular overlayer. To quantitatively and simultaneously model the molecular mechanics response of the present system as well as the imaging process by electron tunneling would require the use of a virtual STM machine [25], which in the present case would be prohibitively expensive in terms of computation time.

\section{Conclusions}

In summary, the adsorption and self-assembly of

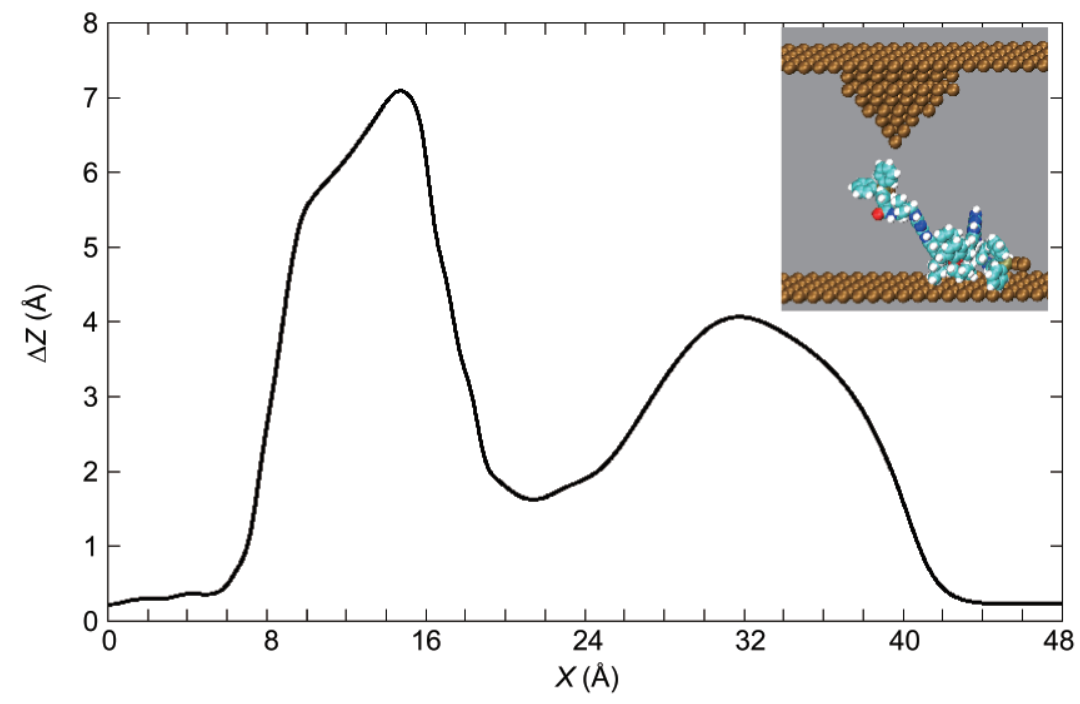

Figure 3 Calculated constant current STM line scan above a single M1 molecule adsorbed on a $\mathrm{Au}(111)$ surface $\left(I_{\mathrm{t}}=1 \mathrm{pA}, V_{\mathrm{t}}=1 \mathrm{~V}\right)$. The tip geometry and the static molecular conformation are shown in the inset. The corrugation $(\Delta Z)$ refers to a situation where the tip apex is in a lateral position next to the molecule. We note that in this situation there may be a contribution to the calculated current from tunneling to the plane of atoms used to support the tip in the modeled geometry 
the hydrogen bonded rosette assembly M2 and its constituent building blocks DEB and M1 on a Au(111) surface have been investigated by UHV-STM. Both individual building blocks were observed to form well-ordered self-assembled structures. When the complete rosette assembly M2 was sublimed, a wellordered surface nanostructure was observed with features indicative of M2 assemblies aligned on the surface in an 'edge-on' manner, The structure is distinctly different from the structures formed when the building blocks are either individually or coadsorbed. Surprisingly, the results therefore suggest that M2 assemblies can be sublimed intact which is an entirely unanticipated result for a supramolecular compound of this size. The stabilization energies for rosette complexes have been investigated in bulk chemistry kinetic studies $[29,30]$ and the melanine-cyanuric acid interaction energy has been determined for molecular gas phase and surface assemblies [19, 31], but direct information about the anticipated stability of the studied rosette complex during vacuum sublimation is difficult to derive due to pronounced solvent effects and uncertainty about possible dissociation mechanisms. The present results imply, however, that the interactions between M2 assemblies are relatively weak while the multiple complementary hydrogen bonds stabilizing the rosette structure have sufficient strength to maintain it intact during both thermal sublimation and the process of landing and organizing on the surface. If it is indeed possible to successfully deposit such a large 3-D hydrogen-bonded supramolecular assembly by normal sublimation methods under UHV conditions, it opens up exciting new avenues for bottomup fabrication of complex 3-D molecular surface nanostructures starting from assemblies synthesized in solution by conventional supramolecular chemistry.

\section{Experimental and theoretical section}

The experiments were performed in a UHV chamber equipped with standard facilities for sample preparation and characterization. Prior to deposition, the single-crystal $\mathrm{Au}(111)$ sample was cleaned by several cycles of $1.5 \mathrm{keV} \mathrm{Ar}^{+}$sputtering followed by annealing at $770 \mathrm{~K}$ for $15 \mathrm{~min}$, resulting in a wellordered herringbone reconstruction. The synthesis of the gold-functionalized rosette assembly (M2) and its building blocks (M1 and DEB) has been described in Refc. [18, 32]. The molecular samples, which existed in powder form, were loaded in a thermal evaporator in the form of a glass tube wound with a metal wire for resistive heating and with a thermocouple pair fused into the glass. The molecules were thoroughly degassed in situ and then thermally sublimed onto a clean $\mathrm{Au}(111)$ sample held at room temperature (RT) using crucible temperatures of $\sim 360 \mathrm{~K}$ (DEB), $\sim 370 \mathrm{~K}$ (M1), and $~ 380 \mathrm{~K}$ (M2), respectively. After deposition, the sample was left at RT for about $10 \mathrm{~min}$ and then transferred in situ to a variable-temperature Aarhus STM [33]. All the STM measurements were carried out in the constant current mode and in the temperature range $100-150 \mathrm{~K}$ to thermally stabilize the resulting surface nanostructures.

The conformation calculations for a single M1 unit were carried out with an extended semi-empirical ASED+ [26] and the STM line scan was calculated with the elastic scattering quantum chemistry technique (ESQC-STM) [27].

\section{Acknowledgements}

We acknowledge financial support from the Danish Ministry for Science, Technology and Innovation through the iNANO Center, the Danish Research Councils, a Marie Curie-Intra-European Fellowship for H. G. (MEIF-CT-2004-010038), the SONS Eurocores program FUN-SMARTS, and the NMP Frontiers European project (NMP4-CT-2004-500328).

\section{References}

[1] Barth, J. V.; Weckesser, J.; Trimarchi, G.; Vladimirova, M.; De Vita, A.; Cai, C. Z.; Brune, H.; Gunter, P.; Kern, K. Stereochemical effects in supramolecular self-assembly at surfaces: 1-D versus 2-D enantiomorphic ordering for PVBA and PEBA on Ag(111). J. Am. Chem. Soc. 2002, 124, 7991-8000.

[2] Xu, W.; Kelly, R. E. A.; Otero, R.; Schöck, M.; Lægsgaard, E.; Stensgaard, I.; Kantorovich, L. N.; Besenbacher, F. Probing the hierarchy of thymine-thymine interactions in 
self-assembled structures by manipulation with scanning tunneling microscopy. Small 2007, 3, 2011-2014.

[3] Otero, R.; Lukas, M.; Kelly, R. E. A.; Xu, W.; Lægsgaard, E.; Stensgaard, I.; Kantorovich, L. N.; Besenbacher, F. Elementary structural motifs in a random network of cytosine adsorbed on a gold(111) surface. Science 2008, 319, 312-315.

[4] Chen, Q.; Richardson, N. Enantiomeric interactions between nucleic acid bases and amino acids on solid surfaces. Nat. Mater. 2003, 2, 324-328.

[5] Theobald, J. A.; Oxtoby, N. S.; Phillips, M. A.; Champness, N. R.; Beton, P. H. Controlling molecular deposition and layer structure with supramolecular surface assemblies. Nature 2003, 424, 1029-1031.

[6] Kong, X. H.; Deng, K.; Yang, Y. L.; Zeng, Q. D.; Wang, C. H-bond switching mediated multiple flexibility in supramolecular host-guest architectures. J. Phys. Chem. C 2007, 111, 17382-17387.

[7] Nath, K. G.; Ivasenko, O.; Miwa, J. A.; Dang, H.; Wuest, J. D.; Nanci, A.; Perepichka, D. F.; Rosei, F. Rational modulation of the periodicity in linear hydrogen-bonded assemblies of trimesic acid on surfaces. J. Am. Chem. Soc. 2006, 128, 4212-4213.

[8] Wintjes, N.; Bonifazi, D.; Cheng, F.; Kiebele, A.; Stöhr, M.; Jung, T.; Spillmann, H.; Diederich, F. A supramolecular multiposition rotary device. Angew. Chem. Int. Ed. 2007, 46, 4089-4092.

[9] Schiffrin, A.; Riemann, A.; Auwärter, W.; Pennec, Y.; Weber-Bargioni, A.; Cvetko, D.; Cossaro, A.; Morgante, A.; Barth, J. V. Zwitterionic self-assembly of L-methionine nanogratings on the $\mathrm{Ag}(111)$ surface. Proc. Natl. Acad. Sci. USA 2007, 104, 5279-5284.

[10] Spillmann, H.; Kiebele, A.; Stohr, M.; Jung, T. A.; Bonifazi, D.; Cheng, F. Y.; Diederich, F. A twodimensional porphyrin-based porous network featuring communicating cavities for the templated complexation of fullerenes. Adv. Mater. 2006, 18, 275-279.

[11] Kelly, R. E. A.; Xu, W.; Lukas, M.; Otero, R.; Mura, M.; Lee, Y.; Lægsgaard, E.; Stensgaard, I.; Kantorovich, L. N.; Besenbacher, F. An investigation into the interactions between self-assembled adenine molecules and a Au(111) surface. Small 2008, 4, 1494-1500.

[12] Schnadt, J.; Rauls, E.; Xu, W.; Vang, R. T.; Knudsen, J.; Laegsgaard, E.; Li, Z.; Hammer, B.; Besenbacher, F. Extended one-dimensional supramolecular assembly on a stepped surface. Phys. Rev. Lett. 2008, 100, 046103.
[13] Barth, J. V.; Costantini, G.; Kern, K. Engineering atomic and molecular nanostructures at surfaces. Nature 2005, 437, 671-679.

[14] De Feyter, S.; De Schryver, F. C. Two-dimensional supramolecular self-assembly probed by scanning tunneling microscopy. Chem. Soc. Rev. 2003, 32, 139150.

[15] Wan, L. J. Fabricating and controlling molecular selforganization at solid surfaces: Studies by scanning tunneling microscopy. Acc. Chem. Res. 2006, 39, 334342.

[16] Otero, R.; Rosei, F.; Besenbacher, F. Scanning tunneling microscopy manipulation of complex organic molecules on solid surfaces. Annu. Rev. Phys. Chem. 2006, 57, 497 -525 .

[17] Prins, L. J.; De Jong, F.; Timmerman, P.; Reinhoudt, D. N. An enantiomerically pure hydrogen-bonded assembly. Nature 2000, 408, 181-184.

[18] Vázquez-Campos, S.; Péter, M.; Dong, M.; Xu, S.; Xu, W.; Gersen, H.; Linderoth, T. R.; Schönherr, H.; Besenbacher, F.; Crego-Calama, M.; Reinhoudt, D. N. Self-organization of gold-containing hydrogen-bonded rosette assemblies on graphite surface. Langmuir 2007, 23, 10294-10298.

[19] Xu, W.; Dong, M.; Gersen, H.; Rauls, E.; VázquezCampos, S.; Crego-Calama, M.; Reinhoudt, D. N.; Stensgaard, I.; Lægsgaard, E.; Linderoth, T. R.; Besenbacher, F. Cyanuric acid and melamine on Au(111): Structure and energetics of hydrogen-bonded networks. Small 2007, 3, 854-858.

[20] Xu, W.; Dong, M.; Vázquez-Campos, S.; Gersen, H.; Lægsgaard, E.; Stensgaard, I.; Crego-Calama, M.; Reinhoudt, D. N.; Linderoth, T. R.; Besenbacher, F. Enhanced stability of large molecules vacuum-sublimated onto $\mathrm{Au}(111)$ achieved by incorporation of coordinated Au-atoms. J. Am. Chem. Soc. 2007, 129, 10624-10625.

[21] Xu, W.; Dong, M.; Gersen, H.; Rauls, E.; VázquezCampos, S.; Crego-Calama, M.; Reinhoudt, D. N.; Lægsgaard, E.; Stensgaard, I.; Linderoth, T. R.; Besenbacher, F. Influence of alkyl side chains on hydrogen-bonded molecular surface nanostructures. Small 2008, 4, 1620-1623.

[22] Mullen, T. J.; Dameron, A. A.; Weiss, P. S. Directed assembly and separation of self-assembled monolayers via electrochemical processing. J. Phys. Chem. B 2006, 110, 14410-14417.

[23] Monnell, J. D.; Stapleton, J. J.; Dirk, S. M.; Reinerth, 
W. A.; Tour, J. M.; Allara, D. L.; Weiss, P. S. Relative conductances of alkaneselenolate and alkanethiolate monolayers on Au\{111\}. J. Phys. Chem. B 2005, 109, 20343-20349.

[24] Kumar, A. S.; Ye, T.; Takami, T.; Yu, B. -C.; Flatt, A. K.; Tour, J. M.; Weiss, P. S. Reversible photo-switching of single azobenzene molecules in controlled nanoscale environments. Nano Lett. 2008, 8, 1644-1648.

[25] Bouju, X.; Joachim, C.; Girard, C.; Tang, H. Mechanics of $(\mathrm{Xe})_{N}$ atomic chains under STM manipulation. Phys. Rev. B 2001, 63, 085415.

[26] Ample, F.; Joachim, C. A semi-empirical study of polyacene molecules adsorbed on a $\mathrm{Cu}(110)$ surface. Surf. Sci. 2006, 600, 3243-3251.

[27] Sautet, P.; Joachim, C. Electronic transmission coefficient for the single-impurity problem in the scattering-matrix approach. Phys Rev. B 1988, 38, 12238-12247.

[28] Pizzagali, L.; Joachim, C.; Bouju, X.; Girard, C. The resistance of a $(\mathrm{Xe})_{n}$ atomic wire. Europhys. Lett. 1997, 38, 97-102

[29] Seto, C. T.; Whitesides, G. M. Synthesis, characterization, and thermodynamic analysis of a $1+1$ self-assembling structure based on the cyanuric acid.cntdot.melamine lattice. J. Am. Chem. Soc. 1993, 115, 1330-1340.

[30] Prins, L. J.; Neuteboom, E. E.; Paraschiv, V.; CregoCalama, M.; Timmerman, P.; Reinhoudt, D. N. Kinetic stabilities of double, tetra-, and hexarosette hydrogenbonded assemblies. J. Org. Chem. 2002, 67, 48084820.

[31] Staniec, P. A.; Perdigão, L. M. A.; Rogers, B. L.; Champness, N. R.; Beton P. H. Honeycomb networks and chiral superstructures formed by cyanuric acid and melamine on Au(111). J. Phys. Chem. C 2007, 111, 886893.

[32] van Manen, H. J.; Paraschiv, V.; Garcia-Lopez, J. J.; Schonherr, H.; Zapotoczny, S.; Vancso, G. J.; CregoCalama, M.; Reinhoudt, D. N. Hydrogen-bonded assemblies as a scaffold for metal-containing nanostructures: From zero to two dimensions. Nano Lett. 2004, 4, 441-446.

[33] Lægsgaard, E.; Osterlund, L.; Thostrup, P.; Rasmussen, P. B.; Stensgaard, I.; Besenbacher, F. A high-pressure scanning tunneling microscope. Rev. Sci. Instrum. 2001, 72, 3537-3542. 